\title{
The impact of information systems on work structure and culture in an emergency department
}

\section{Atlarelang Basetsana Kesiilwe}

School of Computing and Information Systems

The University of Melbourne, Victoria, Australia

Email: akesiilwe@student.unimelb.edu.au

\section{Reeva Lederman}

School of Computing and Information Systems

The University of Melbourne, Victoria, Australia

Email: reeva.lederman@unimelb.edu.au

\section{Suelette Dreyfus}

School of Computing and Information Systems

The University of Melbourne, Victoria, Australia

Email: suelette@unimelb.edu.au

\section{Jonathan Knott,}

Department of Emergency Medicine,

The Royal Melbourne Hospital, Victoria, Australia

Email: J onathan.Knott@mh.org.au

\section{Abstract}

This paper describes a study where consultants in an emergency department will be observed and interviewed with the aim of evaluating the impact of the progressive implementation of an information system in an emergency department in a metropolitan hospital in Australia. The study is the third stage of a 3-stage longitudinal study which is building on data from two previous studies that were conducted in the same setting in 2008 and 2012. The evaluations will be based on predefined task categories to investigate how information systems in health settings affect time and prioritisation of tasks. The study aims to understand how the changes brought by information systems implementation can be managed to fit in with the structure and culture of work in the hospital setting. The outcome of this research will inform guidelines for how information systems for hospitals can be designed and implemented.

Keywords: emergency department, information systems, time-motion studies. 


\section{Introduction}

Emergency medicine provides rapid management of acuteillness and injury. According to the Australian Institute of Health and Welfare, there were 7.8 million presentations across Australian emergency departments (EDs) in the period 2016-2017. This is an increase in comparison to the period 2015-2016 where 7.3 million presentations were reported. The report states that between the periods 2012-2013 and 2015-2016 there was an increase of 3.7\% on average of presentations every year, and between the periods 2015-2016 and 2016-2017, the presentations increased by 3.9\% on average (Emergency Department Care 2016-2017 Australian Government). The increase in ED presentations is due to varying reasons such as the financial burden of primary care (J ones and Thornton 2013), an aging population (Lowthian et al. 2012) and ED visits by people living in lower socio-economic areas (Australian Bureau of Statistics 2014) who cannot access other modes of care. Despite an increase in online care access (Fan et al. 2010; Fan et al. 2013; Fan and Lederman 2018; Lederman et al. 2014), patients under stress still present constantly at the ED. This increase in ED demand leads to complexities such as variation in patient conditions; overcrowding which may lead to longer waiting times, increased mortality rates and a shortage of resources such as beds (Bernstein et al. 2009; Forero et al. 2010; Moskop et al. 2009), which makes the ED workflow non-linear and challenging (Wang 2009).

The practice of medicine in EDs is often compromised by factors such as the need for time-sensitive patient care decisions, the frequent lack of key patient information and the extreme variation in presenting complaints and medical conditions (Hoffman et al. 2013). To overcome the complexities in EDs, information technology (IT) is often integrated into ED processes. This, as stated by Shekelle et al (2006), is due to the fact that information technology use in healthcare is seen as strategic in creating efficiencies and improvements that are cost effective and beneficial to patients. In emergency medicine, information technology is ideal in supporting the collaborative work and information needs of clinicians thus optimising the delivery of better patient care (Berg 2003; Laxmisan et al. 2007; Taylor 2004) in terms of timely treatment, attendance and execution, and the reduction of ED waiting time. As a result, Information Systems (IS) implementation in the ED is increasingly aimed at contributing to a patientcentred approach to care (Lederman and Ben-Assuli 2017; Taylor et al. 2017), to improve clinical outcomes through the provision of a platform for shared multidisciplinary and continuous patient care and a facilitation of communication between healthcare providers (Vezyridis et al. 2014).

\subsection{Context of the study}

The ED is a busy and fast paced environment and information systems (IS) are often implemented to support activities and clinical tasks. The proposed study is the third stage of a 3-stage exploratory longitudinal study (Kee et al. 2012; Lederman et al. 2015; Peng et al. 2010; Peng et al. 2013;) where data collected in the years 2008, 2012 and 2018 will be compared to understand the progressive change in information systems use and deployment in an emergency department.

Before the current EDIS was introduced the ED had a paper-based system and a number of stand-alone computer systems. These systems included the hospital admission system, test result retrieval system and a picture archiving and communications system (PACS) for storing and viewing radiology results. The current system was implemented in 2012 to automate administration, triage and tracking, clinical notes, test ordering and conditional data collection. The system in use is about to be replaced as it is no longer supported in Australia, and a new IS is to be implemented. With this new implementation, there is an expectation of integrating some functions and an opportunity for more flexibility in improving the workflows and information exchange as it captures real time patient data. We intend to analyse its impact compared to collected data from the previous studies discussed below:

\subsubsection{Overview of the 2008 study}

This study aimed at evaluating how consultants spend time on various tasks based on a list of predetermined task categories. These categories are clinical care, transiting, documentation, computer use, communication, pharmacy, non-clinical tasks and communication. When this study was conducted, paper-based patient records were still in use and the EDIS (Emergency Department Information System) was used to document the patient pathway from arrival till consultation. The study was conducted over a period of 2 months with a total of over 130 hours of recorded observations of duty and resuscitation consultants. On each hourly observation, it was recorded that a total of 101 tasks were performed by the consultants. 


\subsubsection{Overview of the 2012 study}

The second study was conducted over a period of 59 days with a total of 400 hours of recorded observations. The observations were based on the structuration theory to determine the patterns of work activities that emerge during the implementation of an information system and quantify the time spent on activities that are involved in direct care of patients and also activities that are involved in indirect patient care. The implementation of the information system as highlighted in the study resulted in changes to work routines: information processing for the different groups of clinicians changed due to the centralisation of information collection, storage and access; task prioritisation became an issue as staff were pressured to make decisions based on competing priorities of patient care quality vs economic demands.

This longitudinal study will provide insights into the impact of changing/updating an IS in an ED environment. The comparison of data from the two studies discussed above revealed that implementation of information systems affects clinical practices in terms of both structure and culture. The intended study is important as it extends the scope of the previous studies beyond the impact of IS on time spent on work activities to examining work routines and assessing how the resulting change is managed. The information system will be examined from a technical dimension - the perceived usefulness of the technology, the social dimension - how the technology fits in with existing working roles and; the organisational dimension - existing workflows (Cresswell and Sheikh 2013). Social consequences that affect the outcomes of IS implementation will also be examined as they reflect the difficulties that stem from the integration of the new technology with existing working practices of users as well as with organisational processes (Cresswell 2016).

\section{Literature Review}

\subsection{The evolution of IS in healthcare}

The application of IS in healthcare has allowed for easy access to patient information, facilitated decision making as well as the support of administrative tasks such as appointments scheduling, registration and discharge of patients (Mohamadali and Zahari 2017).

The healthcare industry has lagged behind the business environment in the introduction of information technology. The use of information systems in medicine is often dated to the 1950s with the work of Robert Ledley, who applied computers of the time to dentistry (and later developed the first computed tomography scanner). The size of computing devices of the time limited their application to clinical practice, but with the development of microcomputers in the 1970s and 1980s, use at the bedside became possible (Hoffman et al. 2013). In the United States the Health Information Technology for Economic and Clinical Health (HITECH) Act was implemented in order to promote meaningful use of information technology - the act was implemented to promote widespread of information technology (Congressional Research Service, 2009); in the UK in 2014, the National Health Service (NHS) launched the five year forward view which aims at improvements in quality and service by harnessing technology and innovation to achieve digitisation in healthcare (Honeyman et al. 2016); in Australia, the Australian Digital Health Agency launched the National Digital Health Strategy which is aimed at achieving outcomes such as improved access to patient information; better availability of information on medications and prescriptions; digitally-enabled models of care that drive improved accessibility, quality, safety and efficiency by the year 2022 (Australian Digital Health Agency).

\subsection{IS Implementation in the ED}

Emergency care involves the provision of short term treatment on patients and efficient performance of complex tasks - most patients that present to the ED are critical patients and examination of patients needs to be performed quickly due to high patient volume (Lenz and Reichert 2007). In recent years, the role of the ED has expanded to also provide primary care which has added to the work load in ED settings. Thus, the implementation of information systems in ED is a complex process due to the multifaceted and reciprocal relation between IS and organization. Often in emergency departments IS implementation is perceived as disruptive to work routines and the workflow. This, as noted by Rodriguez, Aziz, and Chatwin, (2014, p.88) is due to the fact that "emergency departments (EDs) workflows require robust coordination between resources for treatment, referral, admission and discharge purposes in order to maintain a swift and accurate patient flow through the different stages during their ED visit". Information system studies in regard to workflow have shown that users often experience difficulty in integrating the technology in their routine clinical practices, and despite the perceived benefits of the technology, difficulties have been reported in assimilating the use of the 
technology in their work (Callen et al. 2013). These difficulties as stated by the authors are in relation to significant data entry demands required and the time taken and are often seen as contributing to workflow disruptions. IS implementation also changes the patient care processes because the aim of such systems implementations is to promote efficiency (Vartak et al. 2009).

The infusion of health information technologies in ED environments is driven by the need to facilitate communication, promote ease of information flow and workflow and enhance decision making and patient safety (Kane and Luz 2015). It has been reported that health information technologies (HIT) capabilities lead to improvement in ED efficiency by reducing waiting times and improving turnaround times when ordering tests and medications during patient visits (Selck and Decker 2016).

\subsection{IS implementation evaluation}

The implementation of IS, which refers to the process of planning, testing, adopting and integrating IS to ensure the integration of the technology into organisational routine is a complex process which is often met with challenges such a slow implementation process, integration issues between the system and work place practices, (Cresswell and Sheikh 2013). To understand the impact of IS implementation in EDs and gain insights into how IS impacts clinical efficiency, it is imperative that the work processes and workflows are studied (Zheng et al. 2011). As noted by the authors, "to develop such an understanding requires rigorously conducted research that can generate compatible and comparable results to inform effective technology designs and implementation approaches" (Zheng et al. 2011, p. 704). Such studies allow a deeper understanding of IS use by providing insights into IS implementation in EDs. As explained by Jones et al (2012), the IT paradox comprises of three categories being mismeasurement, mismanagement, and poor usability. Therefore, an understanding of information system use can enable the design and implementation of systems that are specific to the unique needs of emergency medicine practitioners thereby promoting usability of the systems and enabling ways of measuring system use.

Reduced costs and improved patient care quality are often the drivers of IS implementation in most healthcare settings (Cresswell et al. 2013). Measurement and prediction of outcomes of IS implementations allow the conceptualisation of the required transformations. Evaluations of IS implementations are often done at the individual level to investigate what drives individuals to adopt IS; at the group level to investigate issues such as resistance towards adoption; and at the organisational level which investigate the readiness of an organisation to adopt IS (Lapointe and Rivard 2007). The need for orderly routines in patient care provision and the need for sensitivity to variation in patient conditions is what characterizes healthcare delivery settings. Often the tension between the routine and the variability in presentations lead to complexities surrounding the implementation and usage of IS as is the case in most ED settings (Agarwal et al. 2011). These authors emphasise the importance of learning to determine the best way to align both technology and organisation to achieve a good fit between the capabilities afforded by the technology and the desired patterns of actual use. The intended study serves as a basis for learning about the implications of the implementation of IS on clinicians' work tasks in terms of quality and speed; and the impact on patient care in terms of the time spent interfacing with patients.

\section{Methodology}

This study will use a time-motion approach (Kesiilwe et al. 2018) to explore the impact of technology use on clinical activities and identify patterns of use to generate findings that can inform the design of IT based interventions for patient care. The evaluation will use a mixed methods case study based approach where both quantitative and qualitative methods will be used for data gathering. The combined use of both the qualitative and quantitative methods in a single evaluation will yield results that a single approach may not yield, and because the limitations of using either method is offset by the other (Creswell and Clark 2011). A mixed methods approach also creates a synergy whereby one method enables the other to be more effective and together both methods would provide a complete understanding of the evaluation (Greene and Caracelli 1997).

A case study research approach will be used as it enables a focus on an intensive assessment of events that occur within the structure of the organisation and the selection of cases is representative of the phenomenon under study (Wynn and Williams 2012). Case study is suitable for this study since it's an exploratory study, the case study methodology as noted by Yen (2003), allows for results to be interpreted directly and allows transferability of solutions and Gomm et al (2000) also advocates that good case studies allow for the recognition of complexity of social truths as well as the generalisability of a solution or instances of that solution. 
Quantitative data will be collected through continuous observations using the "shadowing technique" which allows one person to be observed and studied in detail at a time as is common with time motion studies. Time-motion studies have been conducted in health care settings such as emergency department, general medicine and surgical environment to assess if medical practitioners maximise the time spent on patient care by timing their activities (Hollingsworth et al. 1998). The use of time motion studies have been in existence since the early twenties as a result of a recognition of inefficiencies and a wastage of resources from industrial processes which led to the development of techniques aimed at reducing process times to achieve efficiency (Taylor 1914) and the technique was also adopted in the healthcare industry to assess inefficiencies and promote cost reduction (Baumgart and Neuhauser 2009). The medical staff will be shadowed for a period of time using a tablet that has a specialised time stamp software to capture the activities being performed as well as to time the execution of the activities. This technique has been adopted by a few studies in Australia (Lo et al. 2010; Westbrook et al. 2008;), with the aim to quantify the time medical staff spent in patient care activities. 19 consultants/ senior doctors in the ED will each be observed at random and each observation session will last for $2 \mathrm{hrs}$. Observations will be conducted for the first two weeks of each month and 2 observation sessions will be carried out on week days for a period of 3 months.

Qualitative data will be collected through structured interviews with the same participants that were shadowed. When conducting semi structured interviews 'open, direct, verbal questions will be used to elicit detailed narratives and stories' (DiCicco-Bloom and Crabtree 2006) as cited in Whiting (2008).

\section{Conclusion}

This study is a continuation into stage three of a longitudinal study which evaluates how theintroduction of IS can affect organisational change over time with regards to work structure and time spent on the different tasks relating to patient care. We will reach a deeper understanding than previous studies which have focussed on differences in working hours and habits as opposed to broader socio-logical and cultural issues that affect the ways tasks are carried out. A final goal of the project is to propose a systematic and practical way of managing the implementation of hospital IS for better achievement of intended organisational goals.

\section{References}

Agarwal, R., Gao, G., DesRoches, C., and J ha, A.K. 2011. "The role of information systems in healthcare: Current research and road ahead," Information Systems Research, (22), J uly, pp 419-428.

Emergency department care 2016-17: Australian hospital statistics. https:// www.aihw.gov.au/ reports/ hospitals/ ahs-2016-17-emergency-departmentcare/ contents/table-of-contents. Retrieved 26 July 2018.

Australian Digital Health Agency. https://conversation.digitalhealth.gov.au/australias-nationaldigital-health-strategy Retrieved 26 J uly 2018.

Baumgart, A., and Neuhauser, D. 2009. "Frank and Lillian Gilbreth: scientific management in the operating room," Quality and Safety in Health Care, (18:5), J uly, pp 413-415.

Bernstein, S., Aronsky, D., Duseja, R., Epstein, S., Handel, D., Hwang, U., McCarthy, M., J ohn McConnell, K., Pines, J., Rathlev, N., Schafermeyer, R., Zwemer, F., Schull, M. and Asplin, B. 2009. "The Effect of Emergency Department Crowding on Clinically Oriented Outcomes," Academic Emergency Medicine, (16:1), July, pp.1-10.

Berg, M. 2003. "The search for synergy: interrelating medical work and patient care information systems," Method of Information in Medicine, (42), J une, pp 337- 344.

Cameron, P.A., Bradt, D.A., and Ashby, R. 1996. "Emergency medicine in Australia," Annals of Emergency Medicine, (28:3), July, pp 342-346.

Chaudhry, B., Wang, J., Wu, S., Maglione, M., Mojica, W., Roth, E., Morton, C., and Shekelle, P.G. 2006. "Systematic review: impact of health information technology on quality, efficiency, and costs of medical care," Annals of internal medicine, (144:10), J uly, pp 742-752.

Cresswell, J.W., and Clark, V.L.P. 2011. Designing and Conducting Mixed Methods Research. SAGE Publications Inc, California. 
Cresswell, K. 2016. Evaluation of Implementation of Health IT in: E. Ammenwerth, M. Rigby (eds.), Evidence-Based Health Informatics, Stud Health Technol Inform 222, IOS Press, Amsterdam, 2016.

Cresswell, K.M., Bates, D.W., and Sheikh, A. 2013. "Ten key considerations for the successful implementation and adoption of large-scale health information technology," Journal of the American Medical Informatics Association, (20:e1), October, pp e9-e13.

Cresswell, K., and Sheikh, A. 2013. "Organizational issues in the implementation and adoption of health information technology innovations: An interpretative review," International J ournal of Medical Informatics, (82:5), June, pp e73-e86.

Fan, H., and Lederman, R. 2018. "Online health communities: how do community members build the trust required to adopt information and form close relationships?" European Journal of Information Systems, (27: 1), October, pp 62-89.

Fan, H., Lederman, R., Smith, S., and Chang, S. 2014. "How Trust Is Formed in Online Health Communities: A Process Perspective", Communications of the Association for Information Systems, (34), Article 28.

Fan, H., Lederman, R., Smith, S., and Chang, S. 2010. "Why PeopleTrust in Online Health Communities: An Integrated Approach", 21st Australasian Conference on Information Systems, December 1-3.

Fan, H., Lederman, R., Smith, S., and Chang, S. 2013. "How Online Health Forum Users Assess UserGenerated Content: Mixed-Method Research”, European Conference in Information Systems

Forero, R., Hillman, K., McCarthy, S., Fatovich, D., J oseph, A. and Richardson, D. 2010. "Access block and ED overcrowding," Emergency Medicine Australasia, (22:2), J une, pp 119-135.

Gomm, R., Hammersley, M., and Foster, P (eds.). 2000. Case Study Methods. London: Sage Publications.

Hoffman, J .M., Zorc, J .J ., and Harper, M.B. 2013. “TT in the ED: Past, Present, and Future,” Paediatric Emergency Care, (29:3), J une, pp 402-405.

Honeyman, M., Dunn, P., and McKenna, H. 2016. "A digital NHS? An introduction to the digital agenda and plans for implementation." https:// www.kingsfund.org.uk/publications/digital-nhs Retrieved 26 J uly, 2018.

J ones, P.G. and Thornton, V. 2013. “Does cost drive primary care patients to New Zealand's emergency departments? A systematic review," New Zealand Medical J ournal, (126:1387), J une, pp 15.

Kane, B., and Luz, S. 2015. "Medical teamwork, collaboration and patient-centred care," Behaviour \& Information Technology, (34:6), pp 543-547, DOI: 10.1080/0144929X.2015.1033181 Retrieved August 62018

Kee, R., Knott, J .C., Dreyfus, S., Lederman, R., Milton, S., and J oe, K. 2012. “One hundred tasks an hour: An observational study of emergency department consultant activities", Emergency Medicine Australia, (24:3), October, pp 294-302.

Kesiilwe, A, Lederman, R., Dreyfus, S., and Knott, J . 2018. "A time Motion Study Evaluating the Impact of Information Technology on Emergency Department Consultant Activities," Twenty-Sixth European Conference on Information Systems (ECIS2018), Portsmouth, UK, 2018.

Lapointe, L., and Rivard, S. 2007. "A triple take on information system implementation," Organization Science, (18:1), April, pp 89-107.

Laxmisan, A., Hakimzada, F., Sayan, O.R., Green, R.A., Zhang, J., and Patel, V.L. 2007. "The multitasking clinician: decision-making and cognitive demand during and after team handoffs in emergency care." International J ournal of Medical Informatics, (76:11), J uly, pp 801-811.

Lederman,R., and Ben-Assuli, O. 2017. "Editorial: Patient-centered health information technology: Preparing for a new era." Health, Policy and Technology, (6: 1), October, pp 1-2.

Lederman, R., Fan, H., Smith, S., and Chang, S. 2014. "Who can you trust? Credibility assessment in online health forums" Health, Policy and Technology, (3:1), October, pp 13-25.

Lederman, R., Kurnia, S., Peng, F., and Dreyfus, S. 2015. "Tick a box, any box: a case study on the unintended consequences of system misuse in a hospital emergency department", J ournal of Information Technology Teaching Cases, (5:2) October, pp 74-83. 
Moskop, J.C., Sklar, D.P., Geiderman, J.M., Schears, R.M. and Bookman, K.J. 2009. “Emergency department crowding, part 1-concept, causes, and moral consequences," Annals of Emergency Medicine, (53:5), May, pp 605-611.

Peng, F., R., Kurnia, S., Lederman, R. and Dreyfus, S. 2013. "Exploring the Impact of Information System Introduction: The Case of an Australian Hospital Emergency Department", 46th Hawaii International Conference on System Sciences.

Peng, F., Sharma, R., Kurnia, S., Lederman, R. and Dreyfus, S. 2010. "Organisational culture and organisational impacts of information systems: A review of the empirical literature". 21st Australasian Conference on Information Systems, October, pp 1-12.

Redhead, C.S. 2009. "The Health Information Technology for Economic and Clinical Health (HITECH) Act, The Congressional Research Service." https:// digital.library.unt.edu/ark:/67531/metadc743451/ Retrieved 3 August 2018.

Selck, F.W., and Decker, S.L. 2016. "Health information technology adoption in the emergency department," Health services research, (51:1), J uly, pp 32-47.

Shekelle, P., Morton, S.C., and Keeler, E.B. 2006. "Costs and benefits of health information technology,". Evidence Report/Technology Assessment No. 132. (Prepared by the Southern California Evidence-based Practice Center under Contract No. 290-02-0003.) AHRQ Publication No. 06E006. Rockville, MD: Agency for Healthcare Research and Quality.

Taylor, N., Lederman, R., and Bosua, R. 2017. "Person-centred information for discharge home" The 28 ${ }^{\text {th }}$ Australasian Conference in Information Systems, Hobart, Dec 4-7.

Taylor, T.B. 2004. "Information management in the emergency department." Emergency medicine clinics of North America, (22), J uly, pp 241- 257.

Wang, L. 2009. "An agent-based simulation for workflow in emergency department," In Systems and Information Engineering Design Symposium. SIEDS'09 J uly, pp 19-23

Vartak, S., Crandall, D.K., Brokel, J.M., Wakefield, D.S., and Ward, M.M. 2009. “Professional practice and innovation: transformation of emergency department processes of care with EHR, CPOE, and ER event tracking systems," Health Information Management J ournal, (38:2), October, pp 2732.

Vezyridis, P., Timmons, S., and Wharrad, H. 2011. "Going paperless at the emergency department: a socio-technical study of an information system for patient tracking." International J ournal of Medical Informatics, (80:7), J uly, pp 455-465.

Westbrook, J.I., Duffield, C., Li, L., and Creswick, N.J. 2011. "How much time do nurses have for patients? A longitudinal study quantifying hospital nurses' patterns of task time distribution and interactions with health professionals," BMC Health Services Research, (11:1), pp 319. https:// doi.org/ 10.1186/ 1472-6963-11-319 Retrieved 20 July 2018

Wynn Jr, D., and Williams, C.K. 2012. "Principles for Conducting Critical Realist Case Study Research in Information Systems," MIS Quarterly, (36:3), J uly, pp 787-810.

Zheng, K., Guo, M.H., and Hanauer, D.A. 2011. "Using the time and motion method to study clinical work processes and workflow: methodological inconsistencies and a call for standardized research,"J ournal of the American Medical Informatics Association, (18:5), April, pp 704-710.

\section{Copyright}

Copyright: (c) 2018 authors. This is an open-access article distributed under the terms of the Creative Commons Attribution-NonCommercial 3.0 Australia License, which permits non-commercial use, distribution, and reproduction in any medium, provided the original author and ACIS are credited. 